\title{
Successful treatment of a keratoacanthoma in a young patient with the application of topical $5 \%$ imiquimod cream
}

\author{
Kerasia-Maria Plachouri $^{1}$ | Francesk Mulita ${ }^{2}$ ( ) Dimitrios Bousis $^{3}$ | \\ Levan Tchabashvili $^{2}$ | Elias Liolis ${ }^{3}$ | Charalampos Kaplanis ${ }^{2}$ | Ioannis Perdikaris $^{2}$ | \\ Fotios Iliopoulos $^{2}$ | Georgios-Ioannis Verras ${ }^{2}$ | Vasilios Tolias ${ }^{1}$ | Sophia Georgiou ${ }^{1}$
}

\author{
${ }^{1}$ Department of Dermatology, General \\ University Hospital of Patras, Patras, \\ Greece \\ ${ }^{2}$ Department of General Surgery, \\ General University Hospital of Patras, \\ Patras, Greece \\ ${ }^{3}$ Department of Internal Medicine, \\ General University Hospital of Patras, \\ Patras, Greece

\section{Correspondence} \\ Francesk Mulita, Department of \\ General Surgery, General University \\ Hospital of Patras, Patras, Achaia, \\ Greece. \\ Email: oknarfmulita@hotmail.com \\ Funding information \\ None
}

\begin{abstract}
Keratoacanthomas (KA) are epithelial tumors that present as rapidly evolving nodules with a central hyperkeratotic plug and occasionally show signs of spontaneous regression. A 21-year-old patient strongly refused the diagnostic biopsy and insisted on a nonsurgical treatment. He was successfully treated with imiquimod $5 \%$ cream.
\end{abstract}

\section{K E Y W O R D S}

epithelial tumor, imiquimod cream, keratoacanthomas

\section{CASE DESCRIPTION}

A 21-year-old man presented in our department due to an asymptomatic nodule in the proximal fifth digit of the right hand, that had first appeared 3 months prior to the referral. The lesion rapidly progressed in size during the first 4 weeks and afterward remained stable. The clinical examination revealed a firm skin-colored nodule, of approximately $1.8 \times 1.8 \mathrm{~cm}$ in size, with a central nonremovable keratinous plug (Figure 1A). The clinical diagnosis of keratoacanthoma was made, and a biopsy was recommended for diagnosis confirmation. The patient strongly refused the diagnostic biopsy and insisted on a nonsurgical treatment. We, therefore, suggested a regimen of topical imiquimod 5\% cream under occlusion, for five consecutive days per week, over a period of 4 weeks.
Two weeks after treatment initiation, a prominent local inflammatory reaction could be documented, resulting in crust formation and erosion (Figure 1B). Four weeks after the completion of treatment, the lesion was markedly flattened, until complete resolution was achieved (Figure 1C).

Keratoacanthomas (KA) are epithelial tumors that present as rapidly evolving nodules with a central hyperkeratotic plug and occasionally show signs of spontaneous regression. ${ }^{1}$ The treatment of choice for KAs is surgical excision, as it provides the advantage of complete tumor removal in a short period of time. ${ }^{1,2}$ However, conservative therapeutic strategies are also to be considered. ${ }^{2}$

\section{CONFLICTS OF INTEREST}

There are no conflicts of interest to declare. 

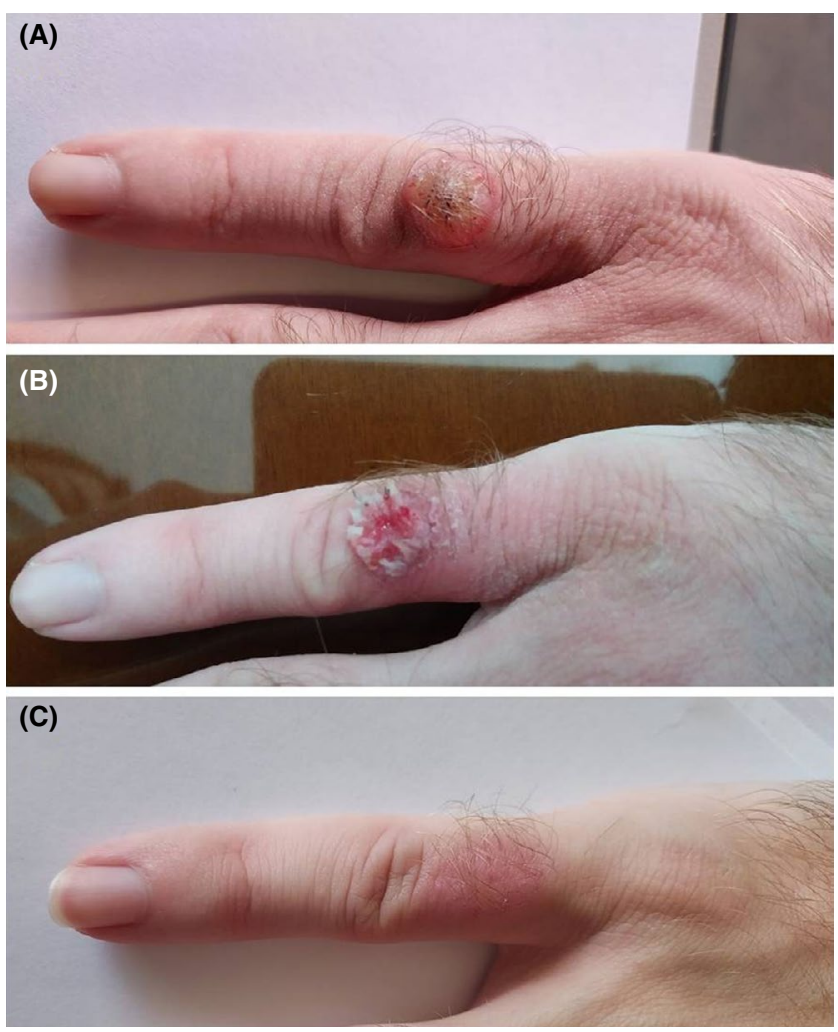

F I G URE 1 A. KA in the proximal fifth digit of the right hand, before treatment B. KA in the proximal fifth digit of the right hand, 3 weeks after treatment initiation $\mathrm{C}$. Complete resolution of the KA in the proximal fifth digit of the right hand, 4 weeks after the completion of treatment

\section{AUTHOR CONTRIBUTIONS}

FM, EL, DB, LT, G-IV, IP, FI, CK, VT, and K-MP contributed to the clinical data collection and prepared the case report. FM, SG, and K-MP contributed to the design of the case report presentation and performed the final revision of the manuscript.

\section{CONSENT}

The written informed consent was obtained from the patient for publication of this case report.

\section{DATA AVAILABILITY STATEMENT}

Data are available on request from the authors.

\section{ORCID}

Francesk Mulita (D) https://orcid. org/0000-0001-7198-2628

\section{REFERENCES}

1. Pancevski G, Pepic S, Idoska S, Tofoski G, Nikolovska S. Topical imiquimod $5 \%$ as a treatment option in solitary facial keratoacanthoma. Open Access Maced J Med Sci. 2018;6:531-535.

2. Janik JP, Bang RH. Traumatic keratoacanthoma arising in a 15-year-old-boy following a motor vehicle accident. Pediatr Dermatol. 2006;23:448-450.

How to cite this article: Plachouri K-M, Mulita F, Bousis D, et al. Successful treatment of a keratoacanthoma in a young patient with the application of topical 5\% imiquimod cream. Clin Case Rep. 2021;9:e04920. https://doi.org/10.1002/ ccr3.4920 